\title{
Pain management in patients with dementia
}

This article was published in the following Dove Press journal:

Clinical Interventions in Aging

31 October 2013

Number of times this article has been viewed

\section{Wilco P Achterberg' \\ Marjoleine JC Pieper ${ }^{2}$ \\ Annelore H van Dalen-Kok' \\ Margot WM de Waal' \\ Bettina S Husebo ${ }^{3}$ \\ Stefan Lautenbacher ${ }^{4}$ \\ Miriam Kunz ${ }^{4}$ \\ Erik JA Scherder ${ }^{5}$ \\ Anne Corbett ${ }^{6}$}

'Department of Public Health and Primary Care, Leiden University Medical Center, Leiden, ${ }^{2}$ Department of General Practice and Elderly Care Medicine, VU University Medical

Center Amsterdam, Amsterdam, The Netherlands; ${ }^{3}$ Department of Global Public Health and Primary Care, University of Bergen, Bergen, Norway; ${ }^{4}$ Physiological Psychology, Otto Friedrich University Bamberg, Bamberg, Germany; ${ }^{5}$ Department of Clinical Neuropsychology, VU University Amsterdam, Amsterdam, The Netherlands; ${ }^{6}$ Wolfson Centre for Age-Related Diseases, King's College London, London, UK
Correspondence: Wilco P Achterberg Department of Public Health and Primary Care, Leiden University Medical Center. Post zone V0-P, PO Box 9600 , 2300 RC Leiden, The Netherlands Tel +3I 7| 5268412

Email w.p.achterberg@lumc.nl
Abstract: There are an estimated 35 million people with dementia across the world, of whom $50 \%$ experience regular pain. Despite this, current assessment and treatment of pain in this patient group are inadequate. In addition to the discomfort and distress caused by pain, it is frequently the underlying cause of behavioral symptoms, which can lead to inappropriate treatment with antipsychotic medications. Pain also contributes to further complications in treatment and care. This review explores four key perspectives of pain management in dementia and makes recommendations for practice and research. The first perspective discussed is the considerable uncertainty within the literature on the impact of dementia neuropathology on pain perception and processing in Alzheimer's disease and other dementias, where white matter lesions and brain atrophy appear to influence the neurobiology of pain. The second perspective considers the assessment of pain in dementia. This is challenging, particularly because of the limited capacity of self-report by these individuals, which means that assessment relies in large part on observational methods. A number of tools are available but the psychometric quality and clinical utility of these are uncertain. The evidence for efficient treatment (the third perspective) with analgesics is also limited, with few statistically well-powered trials. The most promising evidence supports the use of stepped treatment approaches, and indicates the benefit of pain and behavioral interventions on both these important symptoms. The fourth perspective debates further difficulties in pain management due to the lack of sufficient training and education for health care professionals at all levels, where evidence-based guidance is urgently needed. To address the current inadequate management of pain in dementia, a comprehensive approach is needed. This would include an accurate, validated assessment tool that is sensitive to different types of pain and therapeutic effects, supported by better training and support for care staff across all settings.

Keywords: pain assessment, Alzheimer's disease, cognitive impairment, behavior

\section{Introduction}

There are an estimated 35 million people with dementia across the world. Currently, $5 \%$ of people over 65 years old have a diagnosis of dementia, rising to over $50 \%$ in those aged over 90 years. ${ }^{1}$ Demographic changes in the coming decades and the increasingly aging population will lead to a substantial growth in the number of people affected and in the scale of the challenge associated with providing treatment and care. Pain presents a particular challenge in the treatment of dementia. The prevalence of pain, particularly chronic pain, is strongly related to age, hitting the oldest population the hardest, with prevalence rates of $72 \%$ above the age of 85 years. $^{2}$ Given these circumstances, it is clear that pain is probably very common among people with 
dementia; nevertheless, current knowledge is poor, which frequently leads to inappropriate treatment and care.

"Dementia" is defined as a "clinical syndrome due to disease of the brain, usually of a progressive nature, which leads to disturbances of multiple higher cortical functions, including memory, thinking, orientation, comprehension, calculation, learning capacity, language, and judgment."3 The most common cause of dementia is Alzheimer's disease (AD), but vascular dementia (VaD), frontotemporal dementia (FTD), and Lewy body dementia, are also prevalent. In all subtypes of dementia, specific neuropathological changes are responsible for the decline in function. Besides the deleterious effects on cognition, the neuropathology of dementia is responsible for numerous other symptoms, such as behavioral disturbances, psychological problems, and the breakdown of language and communication. These problems have been summarized as "behavioral and psychological symptoms of dementia" (BPSD). Although memory dysfunction is the best-known symptom, BPSD, along with physical dysfunctions, have the highest impact on quality of life, and are one of the most important reasons for seeking help and institutionalization. ${ }^{4}$ Pain in dementia is also often expressed through behavioral disturbances. In fact, pain is thought to be one of the most important causal factors of BPSD. ${ }^{5}$ However, this causal link is often difficult to identify due to the complexities of BPSD, which change over the stages of dementia and are more frequent in the later stages of the disease. ${ }^{6}$ BPSD arising as a result of pain, such as agitation and aggression, can be extremely distressing for both the individual and their caregiver, and can lead to the inappropriate prescribing of antipsychotic medication instead of adequate pain treatment. While these medications do have their place in the treatment of severe or persistent psychiatric symptoms, they are associated with substantial side effects including increased mortality, cerebrovascular events, and falls. ${ }^{7,8}$

A further important and often forgotten issue is the impact of the neuropathological changes in dementia on pain perception. ${ }^{9}$ The symptomology of dementia also means that assessment of pain is particularly challenging due to the loss of communication ability, which usually occurs during the condition. As a result, commonly used assessment tools are neither valid nor reliable and are difficult to use. To compound this, educational and organizational shortcomings in dementia care settings often hamper the quality of care and treatment, including management of pain.

This narrative review discusses the evidence from relevant and recent literature regarding the challenges of pain management in dementia. The review focuses on four main perspectives that are critical to this discussion (Figure 1).

A literature search performed in PubMed (Medline) to supplement this review identified 1,669 publications relating to pain management in dementia. While the first mention of pain as a probable symptom in dementia appears in a publication in $1989,{ }^{10}$ the first review was not published until $1996,{ }^{11}$ which indicates that scientific interest in this theme is relatively new.

\section{Biological perspective: the effect and consequences of neuropathological changes in dementia on pain}

Both neuropathological and neuroimaging studies have described interconnected brain areas that are important in the mediation of pain processing. , $^{911,12}$ Most studies describe two neuronal networks, the medial and lateral pain systems. The medial pain system - comprising the amygdala, medial thalamus, hippocampus, anterior cortex cinguli, and prefrontal cortex - is a pathway that mediates cognitiveevaluative and motivational-affective aspects of pain. In addition, autonomic-endocrine aspects are also mediated by the medial system., 913 The lateral pain system comprises, among others, the primary somato-sensoric areas and the lateral thalamic nuclei. The sensory-discriminative aspects (localization, intensity, and quality of pain) are mediated by the lateral pain system. ${ }^{9}$ Overlap of the two systems might occur in the insula. Recently, the existence of a third pathway mediating other critical aspects of pain has been proposed. This is thought to be a rostral, or limbic, pain system, which mediates behavioral aspects of pain - for example, agitated behavior as a reaction to pain. ${ }^{14}$

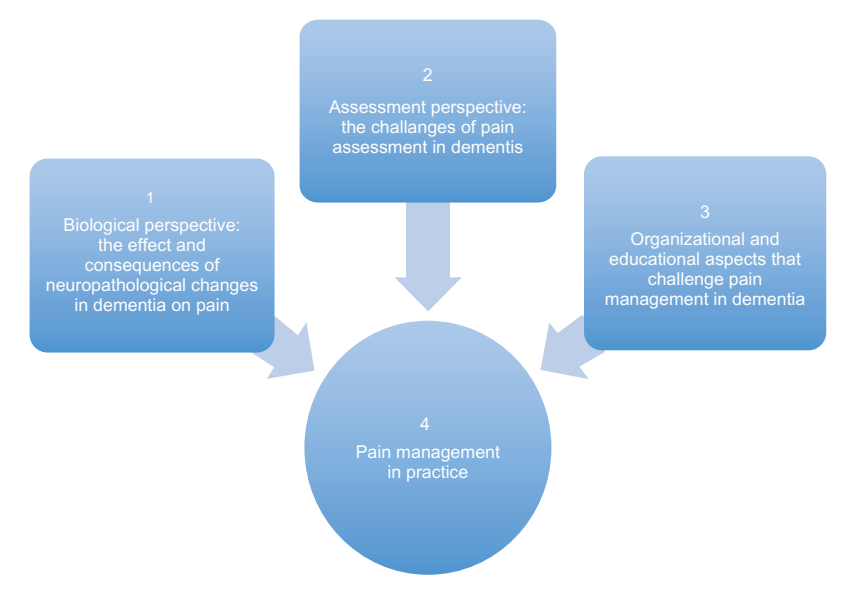

Figure I A model of challenges in pain management in patients with dementia. 


\section{Pain in AD}

In $\mathrm{AD}$, the distribution of neuropathological changes leads to a greater impact on the medial pain system than on the lateral system. This would imply that the cognitive-evaluative and motivational-affective aspects of pain are more greatly affected than the sensory-discriminative aspects. ${ }^{9}$ The clinical consequences for people with $\mathrm{AD}$ would be an unchanged pain threshold but a higher pain tolerance. Some experimental studies have indeed confirmed this theory. ${ }^{15,16}$ As would be expected following examination of the autonomic-endocrine aspects of the medial system and the changes in $\mathrm{AD}$, blunted autonomic responses to pain have also been reported in experimental studies ${ }^{16}$ although these responses are thought to remain active in cases of intense pain. ${ }^{17}$ Interestingly, however, more recent findings have shown that pain processing - as indicated by brain responses in electroencephalography and functional magnetic resonance imaging (fMRI) studies, pain reflexes, and facial responses to noxious stimuli - does not appear to be diminished in Alzheimer patients. Indeed, in some cases, it appears to be elevated. ${ }^{12,18}$ These findings emphasize the caution that must be taken when extrapolating outcomes of animal studies to humans.

The rostral pain system overlaps with several components of the medial and lateral pathways, with the exception of the ventral striatum, which is generally not seen as a part of these other pathways. The striatum is severely affected in AD. Based on fMRI findings, it is hypothesized that people with mild to moderate $A D$ have a relatively increased activation of the striatum in response to pain. ${ }^{14}$ Conversely, this work indicates that there is a relatively decreased activation in severe AD. Behavioral changes in mild and moderate $\mathrm{AD}$ are therefore thought to be stronger, while in severe AD they might be normal or even blunted. ${ }^{14}$ In fact, some clinical studies have found less pain-related behavior in more severely cognitively impaired patients. ${ }^{19,20}$ Relatively strong associations have been shown between pain and depression, as well as unspecified behavioral problems. ${ }^{21,22}$ Associations of pain with agitation, aggression, delusions, wandering, and resistance to care have also been established, although the link is less consistent. ${ }^{23-26}$

In another fMRI study, a connectivity analysis was used to examine the impact of $\mathrm{AD}$ on the integrated functioning of brain regions mediating the sensory, emotional, and cognitive aspects of pain. Functional connectivity between the cortical and subcortical brain regions appeared enhanced in AD patients. Three functionally connected nodes were the right dorsolateral prefrontal cortex, hypothalamus, and periaqueductal gray, which tended to be constantly activated in the AD patients, who received repeated pain stimuli and could not reduce generalized brain activity. ${ }^{27}$ Another important aspect of the neuropathological change that occurs in the prefrontal lobe in people with $\mathrm{AD}$ is the alteration of response to analgesic medication. An experimental study showed that the endogenous expectation and placebo mechanism, an important aspect of pain management, is reduced in people with $\mathrm{AD}$. This effect is particularly pronounced where damage in the connectivity between the prefrontal lobes and the rest of the brain is extensive, or where frontal neuropsychological function, as tested by the Frontal Assessment Battery, is significantly reduced. ${ }^{15}$ It is therefore likely that people with $\mathrm{AD}$ require a higher dosage of pain medication, to achieve the analgesic result that would normally be expected in a cognitively healthy adult. Further, there remains a great deal of uncertainty as to whether changes in the blood-brain barrier that occur during the dementia process might influence the effect of centrally acting pain medication such as morphine. ${ }^{28}$

\section{Pain in other types of dementia}

There have been few studies on pain in different subtypes of dementia such as VaD, FTD, or Lewy body dementia. In VaD, white matter lesions lead to several disconnections between areas of the brain in a process known as "deafferentiation." This is thought to be responsible for an increase in the motivational-affective aspects of pain. This type of pain, also called "central neuropathic pain," occurs frequently in patients who have had a stroke ${ }^{29}$ and there is some clinical evidence that this type of deafferentiation pain might also occur in $\mathrm{VaD} .^{17,30}$

In people with FTD, it is plausible that the atrophy in the prefrontal cortex that characterizes the condition leads to a decrease in the motivational-affective aspects of pain, in a similar way to AD. In one study, patients with FTD reported less pain than patients with $\mathrm{AD}$ following the same experimental pain stimulus. ${ }^{31}$ An underlying mechanism for this differential response may be due to the more extensive pathology in the prefrontal cortex in FTD compared with in AD. Previous reviews and literature consistently highlight the lack of differential evidence around brain pathology and pain experience in different types of dementia. ${ }^{32}$ However, while this criticism is valid, it is important to note that most people with dementia have mixed pathologies. It is particularly common to encounter combinations of gray matter atrophy and white matter lesions, and recent studies have shown that vascular damage, and, consequently, white matter lesions, is a prominent neuropathological characteristic in AD. It is therefore perhaps less useful to consider 
the specific pathologies of pain in different, yet overlapping, types of dementia, and more helpful to consider the locations within the brain that are affected.

\section{Summary}

There is conflicting evidence from neuropathological, neuroimaging, experimental, and clinical research regarding the impact of dementia neuropathology on pain processing and perception. One might speculate that atrophy of gray matter appears to lead to an increase in pain tolerance, while white matter lesions result in a decrease in tolerance. However, the consequences of the disturbed balance in excitatory and inhibitory processes in central nociception are still far from clear. These alterations in pain processing may have significant consequences for pain assessment and treatment, and should be considered when developing pain management approaches for use in dementia. Importantly, the direction of the impact of neuropathology may differ in subtypes of dementia, and even within individuals. There thus remains a great deal of uncertainty regarding the effects of neuropathological changes in dementia. This lack of clarity likely contributes to indecision in practice and to inappropriate treatment choices.

\section{Assessment perspective: the challenges of pain assessment in dementia}

Accurate assessment of pain is a major prerequisite for adequate pain management and to assess the (positive) impact and potential adverse effects of analgesic medications. Assessment of pain in people with dementia is particularly challenging due to the loss of communication ability inherent in the symptomology of the condition, which limits the subjective reporting of pain that would normally be expected with cognitively healthy adults. The examination of a patient in pain aims to clarify the causal and maintaining factors leading to pain, which may be somatic or psychic, or an interaction of both. The outcome of an examination may therefore be the identification of dominating sources or mechanisms of pain, like nociceptive (ie, musculoskeletal), visceral (ie, internal organs), neuropathic (ie, diabetic neuropathy), functional, or psychosomatic (ie, fibromyalgia) pain. Evidence indicates that around $60 \%-80 \%$ of people with dementia in care homes regularly experience pain, most commonly related to musculoskeletal, gastrointestinal and cardiac conditions; genitourinary infections; and pressure ulcers. ${ }^{5}$ Orofacial pain is also of frequent occurrence. ${ }^{33}$ Different forms of pain present different challenges. Pain related to the internal organs, head, and skin is particularly challenging to detect compared with pain related to the musculoskeletal system, which can be identified through gentle guided movements. ${ }^{34}$ Acute pain, such as that following a fall or acute heart attack is easier to assess than chronic pain, which often provokes pain avoidance through reduced movement or relieving posture.

\section{Assessment through self-report}

In the earlier stages of dementia, when cognitive impairment is limited and communication ability is mostly intact, selfreport of pain is usually possible. There are several self-report scales, among which the Visual Analog Scale (VAS), the Numerical Rating Scale, and the Faces Pain Scale (FPS) ${ }^{5}$ are the most frequently used. A study in 129 patients with severe dementia (mini-mental state examination score $<11$ ), which aimed to assess the performance of self-assessment scales (the verbal-, visual-, and faces pain scales), found that $61 \%$ understood at least one scale, ${ }^{35}$ that is, they were able to explain the scale use and correctly indicate positions for no pain and extreme pain on two separate occasions. However, the study found that participants had difficulty using the FPS, which is perhaps less useful, even in earlier stages of dementia. ${ }^{36}$ The "matching of a line length" to the intensity of pain, as required by the VAS, has also been shown to be challenging for people with cognitive impairment. Therefore, simple verbal or numerical categorical scales are recommended. As the neuropathological damage progresses, assessment by self-report becomes more difficult.

In more advanced stages of dementia, the majority of individuals are no longer able to give valid self-reports. In addition to their loss of communication, people are often no longer able to use introspection to gain knowledge about pain, are unable to report or anticipate its onset and duration, and are unable to understand questions related to the evaluation of their pain. ${ }^{37}$ In these individuals, self-report is not an option, and a proxy rater, usually a primary caregiver, who knows the patient and their usual behavior, should be included in pain assessment. However, it should be noted that one should always make an effort to obtain some sort of self-report within the limitations of the individual's symptoms and condition.

\section{Assessment through observation of behavior}

Where self-report is not possible, observation and detection of pain-related behavior is a valuable approach to identification of pain in dementia. An expert panel convened by the American Geriatrics Society (AGS) published guidance outlining the various behavioral expressions of pain in the elderly, including facial expressions, body movements and 
vocalizations, which are helpful when developing assessment tools for dementia (Table 1). ${ }^{38}$ Facial expressions are particularly useful in detecting discomfort in AD. ${ }^{39,40}$ Interestingly, sensory and affective components of pain can be differentially expressed in the face, with sensory aspects shown by movements around the eyes, and affective aspects depicted by movements of the eyebrows and the upper lip. ${ }^{41}$ However, it should be noted that the accurate application of the method of reading facial expressions using the Facial Acting Coding System requires comprehensive training, which may make this approach unfeasible in clinical practice. ${ }^{42}$

Several observational scales have been developed based on the presence or alteration of the behaviors, emotions, interactions, and facial expressions described by the AGS Panel. Several review articles discuss the psychometric properties of these instruments and their use in clinical practice. ${ }^{5,33,42-47}$ A common conclusion of the current body of literature is that there are a number of promising pain assessment instruments available but that most of these require further validation in people with dementia and assessment of their utility in clinical settings. Other weaknesses of many of the existing instruments are that the distinction between

Table I Common pain behaviors in cognitively impaired elderly persons according to the AGS Panel on persistent pain in older persons $^{38}$

I. Facial expressions

Slight frown; sad, frightened face Grimacing, wrinkled forehead Closed or tightened eyes Any distorted expression Rapid blinking

2. Verbalizations, vocalizations

3. Body movements interactions

5. Changes in activity patterns or routines
Sighing, moaning, groaning

Grunting, chanting, calling out

Noisy breathing

Asking for help

Verbally abusive

Rigid, tense body posture, guarding

Fidgeting

Increased pacing, rocking

Restricted movement

Gait or mobility changes

Aggressive, combative, resisting care

Decreased social interactions

Socially inappropriate, disruptive

Withdrawn

Refusing food, appetite change

Increase in rest periods

Sleep, rest pattern changes

Sudden cessation of common routines

Increased wandering

Crying or tears

Increased confusion

Irritability or distress chronic and acute pain is rarely considered; validity studies in several situations where pain might arise, such as at rest, during day-to-day activities, and during guided movements, are often lacking; and it is unclear if different types of pain (nociceptive, neuropathic, visceral) can be addressed. Further, specific conditions such as orofacial pain have been almost completely overlooked..$^{33}$ Given the elevated level of facial response to pain stimuli in people with dementia compared with in cognitively healthy older adults, this is a key omission in the existing tools. ${ }^{18}$

\section{Assessment of neuropathic pain}

Neuropathic pain is often based on underlying diseases such as diabetic neuropathy, after stroke and amputation. Assessing this form of pain in dementia is extremely challenging. The assessment of "central neuropathic pain," which is defined as pain caused by a lesion, or dysfunction of the central nervous system, is even more complex..$^{48}$ Approximately $35 \%$ of stroke patients suffer from post-stroke central neuropathic pain. ${ }^{29}$ Because this deafferentiation also takes place in $\mathrm{VaD}$, it has been suggested that central neuropathic pain is by far the most undertreated type of pain in patients with dementia. ${ }^{49}$ The assessment and treatment of this type of pain is of high clinical relevance, but it has hardly been described in the literature, most likely because it requires assessment and treatment approaches that differ from those of other types of pain. In 2004, the European Federation of Neurological Societies (EFNS) Panel on Neuropathic Pain published guidelines on neuropathic pain assessment that included thorough sensory bedside testing in individuals with neuropathic pain..$^{50}$ This guidance would provide a useful basis for an assessment tool for neuropathic pain. However, as far as the authors are aware, no such instrument has been developed to date.

\section{Organizational and educational aspects that challenge pain management in dementia}

The challenges inherent in the assessment of pain in people with dementia, due to both symptomology and neuropathology, mean that health care workers are not sufficiently prepared to handle the difficulties in establishing good pain management practice for these patients. The literature suggests that a large proportion of these issues could be overcome through better education on specific aspects of pain management and through more effective facilitation of pain assessment within organizations. It has long been established that inaccurate beliefs and poor knowledge 
and training of staff and management in long-term care are important barriers to high quality care. Even experienced staff would be expected to benefit from specific education and training in pain assessment, pharmacological treatment, pain neurophysiology, and non-pharmacological treatments. A major educational goal is to improve their competency in distinguishing pain behaviors from other behavioral symptoms. ${ }^{51}$ Managers in long-term care are often unaware of the best ways to manage pain in people with dementia. Many do not base decisions on evidence-based guidelines and often hold outdated beliefs regarding the use of treatment options (eg, opioid analgesics). ${ }^{52}$ Good-quality training is essential to address this. One recent study showed that after three interactive 3-hour sessions, gaps in staff knowledge of pain management were reduced and pain management strategies were put into practice four times more frequently than after the control intervention. ${ }^{53}$ A controlled pre-post design trial studied the implementation of a pain protocol with a multifaceted approach. Next to skills training and education, this included a pain team and other quality improvement activities. Both quantitative and qualitative evaluation showed that this intervention was successful. ${ }^{54}$

Recommendations to improve pain assessment and management in nursing homes, including national guidelines, have stressed the importance of a well-trained, knowledgeable pain team. ${ }^{55,56}$ In addition, implementation of treatment algorithms and consultation, continuous education, and team building within the care team are seen as the cornerstones of better pain management (Table 2). ${ }^{56}$ A Canadian study that consulted frontline staff and administrators in long-term care revealed overall a general attitude that is open to change in which staff acknowledged the need for better implementation of pain management. Stakeholders identified a number of barriers including a lack of resources and lack of support from funding bodies. Free evidence-based tools and best practices for nurses, who work in nursing homes, are available through www.geriatricpain.org. However, it is clear that to elicit change in practice it will be key to position an accountable professional or onsite leader to champion implementation of better care standards. ${ }^{57}$

Use of evidence-based observational assessment instruments has often been advocated for regular practice. ${ }^{44,55,58}$ Although there is considerable room for improvement in existing instruments, their use is certainly still recommended and can support better and more timely treatment of pain, particularly when self-report is not possible. A critical step in improving pain management is the promotion and implementation of these existing tools. Current
Table 2 Recommendations to improve pain assessment and management in nursing homes ${ }^{56}$

I. Include an initial needs assessment of current pain care practices, formation of a pain quality improvement team guided by a systematic implementation process model, identification of clear quality indicators, and an ongoing educational component

2. Use evidenced-based clinical decision-making algorithms for assessing and treating pain in persons with dementia

3. Collaboratively engage all members of the care team, including residents, nurses at all levels within the organization, prescribers, medical directors, direct care workers, pharmacists, and families when considering pain care process changes

4. Specifically target team-building with a goal of facilitating improvements in communication between prescribers and nurses about pain care in particular

5. Incorporate a plan for regular periodic evaluation of pain management processes (eg, documentation of pain assessments and administration of analgesic medications on a scheduled basis) and resident outcomes, particularly pain severity and satisfaction, into efforts to ensure ongoing implementation of new practices

6. Use consultants with expertise in pain management and process improvement strategies for on-site consultation

uptake and use of instruments is low, and in some cases appears nonexistent. For example, a recent study in acute care settings in Finland showed very low use of pain instruments following hip fracture surgery. When an instrument was used, it was usually the VAS, which is known to provide unreliable information in people with dementia. ${ }^{59}$ Compliance in the use of these observational instruments in long-term care settings has also been disappointing. It is important to emphasize that while implementation of these observational scales is an important step to improve pain management, this alone will not necessarily mean that treatment will be improved. Key evidence and guidance are needed to support the decision-making process to translate a pain score to treatment. This is a complex process, as a recent Dutch study has shown, ${ }^{60,61}$ and several studies emphasize that recognition of the pain does not necessarily lead to appropriate treatment. ${ }^{62-66}$

\section{Pain management in practice}

Some studies have suggested that pain is less prevalent in patients with dementia because they suffer from less comorbidity, ${ }^{67}$ although several other studies have found that people with dementia do not have less painful conditions. ${ }^{68,69}$ Taken together, the literature indicates that about $50 \%$ of patients with dementia are regularly in pain. ${ }^{5}$ The largest study, which included over 5,000 home-care patients, also found no difference in pain prevalence in patients with or without dementia. ${ }^{70}$ 
Pain in people with $\mathrm{VaD}$ has received little attention in research. One of the few studies shows that, in line with the theory based on the neuropathological changes, more specifically white matter lesions, people with possible $\mathrm{VaD}$ may experience an increase in the experience of the motivationalaffective aspects of pain. ${ }^{71}$ Cross-sectional analyses in people with dementia living in nursing homes have demonstrated that there is a particular risk of severe pain in people with severe dementia and a mixed form of dementia (ADVaD) due to the restricted use of pain medication. ${ }^{72}$ Those with ADVaD receiving opioids as pain treatment tended to have higher pain intensity than people without dementia receiving the same treatment. In addition, ADVaD patients have a significantly higher frequency of International Statistical Classification of Diseases and Related Health Problems 10th Revision (ICD-10) diagnoses and are therefore suggested to be more vulnerable. As a consequence, they may have a lower tolerance for opioids. The evidence thus supports the importance of particular caution by physicians when prescribing opioids in people with $\mathrm{ADVaD}$.

International epidemiological research has shown that the elderly in general, but especially those with dementia, receive less pain medication than their cognitively healthy counterparts, even in the same painful situations - for example, after a hip fracture. ${ }^{73}$ The low dosage of pain medication seems to occur consistently in residential, nursing home, and hospital care. ${ }^{63,74-77}$ Remarkably, recently, a few studies have reported a possible overuse of analgesics, particularly paracetamol, in patients with dementia, ${ }^{68,78,79}$ stressing the clinical difficulties and uncertainties in the assessment of pain in these individuals (Figure 2). However, when people with dementia are prescribed pain medication, it is generally of low dosage and stronger pain medication such as opioids, are less likely to be considered. ${ }^{5}$ For instance, patients with a hip fracture who have dementia receive significantly less opioids, both pre- and post-surgery. Where opioids are prescribed, they are used at a dosage that is one-third of that used in cognitively intact persons. ${ }^{73}$

The insufficient management of pain in patients with dementia can be explained by several factors. This uncertainty is partly due to the scarcity of pharmacological studies, which limits understanding of the pharmacodynamics of analgesic medication in this group of people. ${ }^{80}$ The optimal treatment in these patients is therefore predominantly experience based. Clinicians must make decisions on type and dosage of analgesia without clear knowledge of the impact of the cognitive comorbidity of their patient. This lack of knowledge extends among the range of health professionals who work with people with dementia, including nurses and pharmacists. ${ }^{81}$ It is likely that this results in both under- and overtreatment. Efficacy studies of analgesics in patients with dementia are challenging but feasible and there is an urgent need for more research in this area. ${ }^{82}$

The fact that pain is often expressed through challenging behavior, particularly in advanced dementia, has led to several studies investigating the benefit of interventions for both pain and behavior on reducing behavioral symptoms as a proxy measure for pain. Available evidence suggests

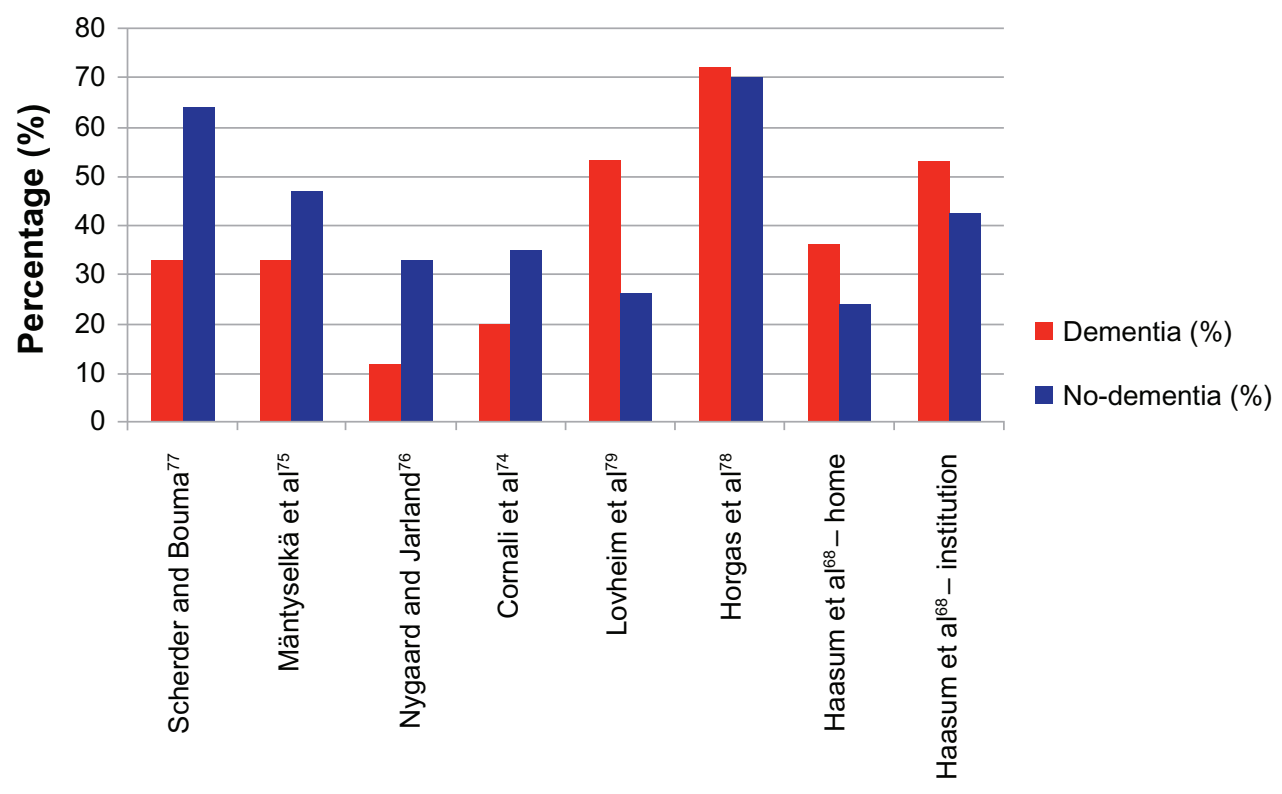

Figure 2 Studies on the prevalence (in \%) of analgesic use in patients with dementia compared with in cognitively unimpaired patients (no dementia). 
that pain interventions targeting behavioral disturbances and behavioral interventions targeting pain are effective in reducing both pain and behavioral symptoms in dementia. ${ }^{83}$ Since 2003, five randomized controlled trials (RCTs) have investigated the treatment effect on pain intensity or behavioral disturbances in these individuals. Manfredi et al evaluated the effect of opioid analgesics on behavioral disturbances in 25 patients with agitation assessed by Cohen-Mansfield Agitation Inventory. ${ }^{84}$ Of the 25 subjects, 13 aged over 85 years showed significant reduction of agitation after 4 weeks. In another 4 -week placebo-controlled crossover study, 39 patients with pain received regular paracetamol. ${ }^{85}$ Pain was assessed using the Discomfort Scale for Dementia of the Alzheimer's Type. No significant differences in pain scores were found in the intervention group. However, the paracetamol dosage was low and might have been insufficient to have a therapeutic effect. In a placebo-controlled crossover trial with 25 patients, Chibnall et al investigated the efficacy of paracetamol on emotional well-being and behavior assessed by Dementia Care Mapping and Cohen-Mansfield Agitation Inventory, respectively. ${ }^{86}$ The study reported significant improvement in activities but found no effect on agitation. In the fourth study, 114 patients with behavioral disturbances were assigned randomly to either a serial trial intervention (STI) of stepped assessment and treatment or usual care. Patients randomized to the STI underwent non-pharmacological comfort intervention. Those still in pain after this treatment $(n=26)$ received analgesics. Pain was assessed using the Discomfort Scale for Dementia of the Alzheimer's Type and behavioral disturbances by the Behavioral Pathology in Alzheimer's Disease Scale. ${ }^{87}$ Results indicate that the STI approach improved behavioral symptoms significantly, but the effect of analgesics is not reported.

It is clear that most of these studies were underpowered with small sample sizes, were restricted to the use of paracetamol or opioids, and lacked validated outcome measures of pain. ${ }^{83}$ The most striking study, an RCT in nursing home patients with dementia and high levels of behavioral symptoms, showed a significant relationship between improvement in agitation and improvement in pain, suggesting that better pain management was the main therapeutic factor. In addition, agitation worsened when the analgesia was discontinued, even though the study continued for only another 4 weeks. ${ }^{88}$ The vast majority of participants in the pain treatment group received only paracetamol, so it is unlikely that the effect was merely due to nonspecific sedation from stronger analgesics. Secondary analyses found that verbal agitation behaviors such as complaining, negativism, repetitious sentences and questions, constant request for attention, and cursing or verbal aggression responded to pain treatment. In addition, restlessness and pacing were sensitive to analgesics. ${ }^{89}$

\section{Evaluation of pain management: responsiveness}

The assessment of pain is the prerequisite for appropriate pain treatment. To provide effective treatment, it is also essential to identify when a treatment response is present. To enable this, there is an urgent need for a pain assessment instrument that can detect changes in pain intensity following treatment. As stated by Cohen-Mansfield and Jensen,,${ }^{90}$ the utility of a pain assessment tool lies in its ability to identify persons whose manifestation of pain will decrease after receiving pain treatment. "Responsiveness" has recently been defined as "the ability of an instrument to detect change over time in the construct to be measured." ${ }^{91}$ As pain is a subjective experience, this measurement requirement is difficult to document in patients with dementia and therefore merits particular attention during development of pain assessment tools. To date, as far as the authors are aware, only two studies have investigated the responsiveness of pain assessment instruments for patients with dementia and nonverbally communicating elderly people. ${ }^{92,93}$ Cohen-Mansfield and Jensen compared the responsiveness of 12 self-report, informant rating, and observational instruments to pain treatment with non-opioids and opioids. Most sensitive to the effect of treatment were the Pain Assessment for the Dementing Elderly and Pain Assessment Instrument in Noncommunicative Elderly tools. Another subsequent trial of pain treatment in nonverbally communicating elderly reported very good responsiveness of the Elderly Pain Caring Assessment 2 tool after the pain treatment with non-opioids of 32 participants with dementia. ${ }^{93}$

To perform valid responsiveness studies, RCTs with appropriate sample sizes are a prerequisite, but most of the current controlled studies did not include a representative sample of elderly with dementia. ${ }^{5}$ Further, it is vital that the final evaluation of the psychometric qualities of a scale considers the criterion of responsiveness against the criterion of reliability. Focusing only on the volatile and state-like aspects of pain (eg, transient facial responses) in an instrument may increase its responsiveness, because every change is detected, but may neglect resistant and trait-like pain features (eg, ongoing relieving posture). The result 
might be a premature "all-clear" when pain has not been fully addressed.

\section{Discussion}

The evidence presented in this review on pain management in people with dementia demonstrates the severe lack of effective assessment and treatment across the range of clinical settings. Pain is common among the elderly due to the increased prevalence of age-related conditions like osteoporosis, arthritis, and cardiovascular disease, and this is also true for people with dementia. These individuals appear to experience the intensity and affective component of pain differently than their cognitively intact counterparts do. In addition, the loss of communication ability leads to serious difficulties in detecting pain, particularly in more severe stages of dementia. In these individuals, pain is often also expressed in specific behaviors, such as agitation or withdrawal, that might mimic psychiatric conditions.

The etiology of these BPSD is multifactorial, and includes the neuropathological changes in the brain related to dementia, but also unmet physical and psychological needs, physical illnesses like urinary tract infections, and pain. In many cases, this results in the inappropriate treatment of behavior with antipsychotic medication. Several studies have shown that treatment of pain might indeed decrease these behavioral symptoms. It is therefore of critical importance to improve the recognition and assessment of pain to ensure that patients receive the most appropriate treatment.

One of the main issues in this process is the development of an assessment toolkit that has good psychometric characteristics, can be used in different types of patients with cognitive impairment, is available in many languages, is sensitive to change, is easy to use in different settings, and is feasible and practical for nurses and other users. This task has been taken up in the European Cooperation in Science and Technology's (COST) action, "Pain assessment in patients with impaired cognition, especially dementia," which started in 2011. This 4-year initiative combines the knowledge of experimental and clinical researchers with that of clinical experts with the goal of reducing the fragmentation in international research and striving for international cooperation, bringing together leading researchers from a wide range of scientific disciplines. The major aim is the development of a comprehensive and internationally agreed-on assessment toolkit for older adults, targeting the various subtypes of dementia (see also COST). ${ }^{94}$

Alongside this work, it is essential that implementation and continuous education and training programs be developed, implemented, and evaluated to ensure the effective use of any new tool. These are prerogative steps for better management, but this will not follow automatically. There is a great need to provide support and clear guidance for clinicians and other health professionals, such as pharmacists and nurses who are involved in the treatment and care of people with dementia, to enable them to make informed decisions, and to remove the current reluctance to prescribe effective analgesia for people with dementia. The further introduction of established "pain teams" and opportunities for staff to consult with experts in all dementia care settings to come to collaborative decisions will also be potentially valuable in ensuring future improvements in the effective management of pain in dementia.

\section{Acknowledgments}

The authors acknowledge the support of the COST program for COST Action TD1005.

AC would like to thank the National Institute for Health Research Mental Health Biomedical Research Centre and Dementia Unit at South London and the Maudsley NHS Foundation Trust and Institute of Psychiatry, King's College London.

\section{Disclosure}

The authors report no conflicts of interest in this work.

\section{References}

1. World Health Organization (WHO). Dementia: A Public Health Priority. Geneva: WHO; 2012. Available from: http://apps.who.int/ iris/bitstream/10665/75263/1/9789241564458_eng.pdf. Accessed September 19, 2013.

2. Duncan R, Francis RM, Collerton J, et al. Prevalence of arthritis and joint pain in the oldest old: findings from the Newcastle 85+ study Age Ageing. 2011;40(6):752-755.

3. WHO. International Statistical Classification of Diseases and Related Health Problems 10th Revision. Geneva: WHO; 2010.

4. Holzer S, Warner JP, Iliffe S. Diagnosis and Management of the Patient with Suspected Dementia in Primary Care. Drugs Aging. Epub June 18, 2013.

5. Corbett A, Husebo B, Malcangio M, et al. Assessment and treatment of pain in people with dementia. Nat Rev Neurol. 2012;8(5):264-274.

6. Cohen-Mansfield J, Thein K, Marx MS, Dakheel-Ali M. What are the barriers to performing nonpharmacological interventions for behavioral symptoms in the nursing home? J Am Med Dir Assoc. 2012;13(4): 400-405.

7. Ballard C, Smith J, Husebo B, Aarsland D, Corbett A. The role of pain treatment in managing the behavioural and psychological symptoms of dementia (BPSD). Int J Palliat Nurs. 2011;17(9):420, 422, 424.

8. Briesacher BA, Limcangco MR, Simoni-Wastila L, et al. The quality of antipsychotic drug prescribing in nursing homes. Arch Intern Med. 2005;165(11):1280-1285.

9. Scherder EJ, Sergeant JA, Swaab DF. Pain processing in dementia and its relation to neuropathology. Lancet Neurol. 2003;2(11):677-686.

10. Andersen F, Lysgaard AP. Shouting by elderly patients with dementia. Ugeskr Laeger. 1989;151(50):3394-3395. Danish. 
11. Farrell MJ, Katz B, Helme RD. The impact of dementia on the pain experience. Pain. 1996;67(1):7-15.

12. Cole LJ, Farrell MJ, Duff EP, Barber JB, Egan GF, Gibson SJ. Pain sensitivity and fMRI pain-related brain activity in Alzheimer's disease. Brain. 2006;129(Pt 11):2957-2965.

13. Sewards TV, Sewards MA. The medial pain system: neural representations of the motivational aspect of pain. Brain Res Bull. 2002;59(3): $163-180$.

14. Monroe TB, Gore JC, Chen LM, Mion LC, Cowan RL. Pain in people with Alzheimer disease: potential applications for psychophysical and neurophysiological research. J Geriatr Psychiatry Neurol. 2012;25(4): 240-255.

15. Benedetti F, Arduino C, Costa S, et al. Loss of expectation-related mechanisms in Alzheimer's disease makes analgesic therapies less effective. Pain. 2006;121(1-2):133-144.

16. Rainero I, Vighetti S, Bergamasco B, Pinessi L, Benedetti F. Autonomic responses and pain perception in Alzheimer's disease. Eur J Pain. 2000;4(3):267-274.

17. Plooij B, Swaab D, Scherder E. Autonomic responses to pain in aging and dementia. Rev Neurosci. 2011;22(5):583-589.

18. Kunz M, Mylius V, Scharmann S, Schepelman K, Lautenbacher S. Influence of dementia on multiple components of pain. Eur J Pain. 2009;13(3):317-325.

19. Monroe T, Carter M, Feldt K, Tolley B, Cowan RL. Assessing advanced cancer pain in older adults with dementia at the end-of-life. JAdv Nurs. 2012;68(9):2070-2078

20. Stevenson KM, Brown RL, Dahl JL, Ward SE, Brown MS. The Discomfort Behavior Scale: a measure of discomfort in the cognitively impaired based on the Minimum Data Set 2.0. Res Nurs Health. 2006;29(6): $576-587$.

21. Gruber-Baldini AL, Zimmerman S, Boustani M, Watson LC, Williams CS, Reed PS. Characteristics associated with depression in long-term care residents with dementia. Gerontologist. 2005;45 Spec No 1(1): $50-55$.

22. Leong IY, Nuo TH. Prevalence of pain in nursing home residents with different cognitive and communicative abilities. Clin J Pain. 2007;23(2):119-127.

23. Tosato M, Lukas A, van der Roest HG, et al. Association of pain with behavioral and psychiatric symptoms among nursing home residents with cognitive impairment: results from the SHELTER study. Pain. 2012;153(2):305-310.

24. Ahn H, Horgas A. The relationship between pain and disruptive behaviors in nursing home resident with dementia. BMC Geriatr. 2013;13:14

25. Cipher DJ, Clifford PA. Dementia, pain, depression, behavioral disturbances, and ADLs: toward a comprehensive conceptualization of quality of life in long-term care. Int J Geriatr Psychiatry. 2004;19(8): 741-748.

26. Volicer L, Frijters DH, Van der Steen JT. Relationship between symptoms of depression and agitation in nursing home residents with dementia. Int J Geriatr Psychiatry. 2012;27(7):749-754.

27. Cole LJ, Gavrilescu M, Johnston LA, Gibson SJ, Farrell MJ, Egan GF. The impact of Alzheimer's disease on the functional connectivity between brain regions underlying pain perception. Eur J Pain. 2011;15(6):568. e561-e511.

28. Banks WA. Drug delivery to the brain in Alzheimer's disease: consideration of the blood-brain barrier. Adv Drug Deliv Rev. 2012; 64(7):629-639.

29. Siniscalchi A, Gallelli L, De Sarro G, Malferrari G, Santangelo E. Antiepileptic drugs for central post-stroke pain management. Pharmacol Res. 2012;65(2):171-175.

30. Achterberg WP, Scherder E, Pot AM, Ribbe MW. Cardiovascular risk factors in cognitively impaired nursing home patients: a relationship with pain? Eur J Pain. 2007;11(6):707-710.

31. Bathgate D, Snowden JS, Varma A, Blackshaw A, Neary D. Behaviour in frontotemporal dementia, Alzheimer's disease and vascular dementia. Acta Neurol Scand. 2001;103(6):367-378.
32. Scherder E, Oosterman J, Swaab D, et al. Recent developments in pain in dementia. BMJ. 2005;330(7489):461-464.

33. Lobbezoo F, Weijenberg RA, Scherder EJ. Topical review: orofacial pain in dementia patients. A diagnostic challenge. J Orofac Pain. 2011;25(1):6-14.

34. Husebo BS, Strand LI, Moe-Nilssen R, Husebo SB, Ljunggren AE. Pain in older persons with severe dementia. Psychometric properties of the Mobilization-Observation-Behaviour-Intensity-Dement ia (MOBID-2) Pain Scale in a clinical setting. Scand J Caring Sci. 2010;24(2):380-391.

35. Pautex S, Michon A, Guedira M, et al. Pain in severe dementia: selfassessment or observational scales? J Am Geriatr Soc. 2006;54(7): 1040-1045.

36. Scherder EJ, Bouma A. Visual analogue scales for pain assessment in Alzheimer's disease. Gerontology. 2000;46(1):47-53.

37. McAuliffe L, Brown D, Fetherstonhaugh D. Pain and dementia: an overview of the literature. Int J Older People Nurs. 2012;7(3):219-226.

38. AGS Panel on Persistent Pain in Older Persons. The management of persistent pain in older persons. J Am Geriatr Soc. 2002;50(Suppl 6): S205-S224.

39. Kunz M, Scharmann S, Hemmeter U, Schepelmann K, Lautenbacher S. The facial expression of pain in patients with dementia. Pain. 2007;133(1-3):221-228.

40. Lints-Martindale AC, Hadjistavropoulos T, Barber B, Gibson SJ. A psychophysical investigation of the facial action coding system as an index of pain variability among older adults with and without Alzheimer's disease. Pain Med. 2007;8(8):678-689.

41. Kunz M, Lautenbacher S, LeBlanc N, Rainville P. Are both the sensory and the affective dimensions of pain encoded in the face? Pain. 2012;153(2):350-358.

42. Lautenbacher S, Niewelt BG, Kunz M. Decoding pain from the facial display of patients with dementia: a comparison of professional and nonprofessional observers. Pain Med. 2013;14(4):469-477.

43. Chapman CR. Progress in pain assessment: the cognitively compromised patient. Curr Opin Anaesthesiol. 2008;21(5):610-615.

44. Herr K. Pain assessment strategies in older patients. J Pain. 2011; 12(3 Suppl 1):S3-S13.

45. Herr K, Bjoro K, Decker S. Tools for assessment of pain in nonverbal older adults with dementia: a state-of-the-science review. $J$ Pain Symptom Manage. 2006;31(2):170-192.

46. Husebo BS, Ballard C, Aarsland D. Pain treatment of agitation in patients with dementia: a systematic review. Int $J$ Geriatr Psychiatry. 2011;26(10):1012-1018.

47. Zwakhalen SM, Hamers JP, Abu-Saad HH, Berger MP. Pain in elderly people with severe dementia: a systematic review of behavioural pain assessment tools. BMC Geriatr. 2006;6:3.

48. Merskey H, Bogduk N. Classification of Chronic Pain: Descriptions of Chronic Pain Syndromes and Definitions of Pain Terms. Seattle, WA: IASP Press; 1994.

49. Scherder EJ, Plooij B. Assessment and management of pain, with particular emphasis on central neuropathic pain, in moderate to severe dementia. Drugs Aging. 2012;29(9):701-706.

50. Cruccu G, Sommer C, Anand P, et al. EFNS guidelines on neuropathic pain assessment: revised 2009. Eur J Neurol. 2010;17(8):1010-1018.

51. Tousignant-Laflamme Y, Tousignant M, Lussier D, et al. Educational needs of health care providers working in long-term care facilities with regard to pain management. Pain Res Manag. 2012;17(5):341-346.

52. Barry HE, Parsons C, Peter Passmore A, Hughes CM. An exploration of nursing home managers' knowledge of and attitudes towards the management of pain in residents with dementia. Int J Geriatr Psychiatry. 2012;27(12):1258-1266.

53. Ghandehari OO, Hadjistavropoulos T, Williams J, et al. A controlled investigation of continuing pain education for long-term care staff. Pain Res Manag. 2013;18(1):11-18.

54. Kaasalainen S, Brazil K, Akhtar-Danesh N, et al. The evaluation of an interdisciplinary pain protocol in long term care. J Am Med Dir Assoc. 2012;13(7):664. e661-e668. 
55. Achterberg WP, de Ruiter CM, de Weerd-Spaetgens CM, Geels P, Horikx A, Verduijn MM; Verenso; LOC; Instituut Verantwoord Medicijngebruik; Nederlands Huisartsen Genootschap; Nederlandse Vereniging voor Klinische Geriatrie; Nederlandse Vereniging voor Psychiatrie; Nederlandse Vereniging voor Anesthesiologie; Koninklijke Nederlandse Maatschappij ter bevordering der Pharmacie; Verpleegkundigen en Verzorgenden Nederland; Nederlandse Vereniging voor Fysiotherapie in de Geriatrie; Ergotherapie Nederland; Nederlands Instituut voor Psychologen. [Multidisciplinary guideline 'Recognition and treatment of chronic pain in vulnerable elderly people']. Ned Tijdschr Geneeskd. 2012;155(35):A4606. Dutch.

56. Swafford KL, Miller LL, Tsai PF, Herr KA, Ersek M. Improving the process of pain care in nursing homes: a literature synthesis. $J \mathrm{Am}$ Geriatr Soc. 2009;57(6):1080-1087.

57. Hadjistavropoulos T, Janzen Claude JA, Hadjistavropoulos H, et al. Stakeholder opinions on a transformational model of pain management in long-term care. J Gerontol Nurs. 2011;37(7):40-51.

58. Hadjistavropoulos T, Herr K, Turk DC, et al. An interdisciplinary expert consensus statement on assessment of pain in older persons. Clin J Pain. 2007;23(Suppl 1):S1-S43.

59. Rantala M, Kankkunen P, Kvist T, Hartikainen S. Post-operative pain management practices in patients with dementia - the current situation in Finland. Open Nurs J. 2012;6:71-81.

60. Cohen-Mansfield J. Even with regular use of an observational scale to assess pain among nursing home residents with dementia, pain-relieving interventions are not frequently used. Evid Based Nurs. Epub May 1, 2013.

61. Zwakhalen SM, van't Hof CE, Hamers JP. Systematic pain assessment using an observational scale in nursing home residents with dementia: exploring feasibility and applied interventions. J Clin Nurs. 2012; 21(21-22):3009-3017.

62. Achterberg WP, Pot AM, Scherder EJ, Ribbe MW. Pain in the nursing home: assessment and treatment on different types of care wards. J Pain Symptom Manage. 2007;34(5):480-487.

63. Closs SJ, Barr B, Briggs M. Cognitive status and analgesic provision in nursing home residents. Br J Gen Pract. 2004;54(509): 919-921.

64. Frantsve LM, Kerns RD. Patient-provider interactions in the management of chronic pain: current findings within the context of shared medical decision making. Pain Med. 2007;8(1):25-35.

65. Leone AF, Standoli F, Hirth V. Implementing a pain management program in a long-term care facility using a quality improvement approach. J Am Med Dir Assoc. 2009;10(1):67-73.

66. Turk DC, Okifuji A. What factors affect physicians' decisions to prescribe opioids for chronic noncancer pain patients? Clin J Pain. 1997;13(4):330-336.

67. Wolf-Klein GP, Siverstone FA, Brod MS, et al. Are Alzheimer patients healthier? J Am Geriatr Soc. 1988;36(3):219-224.

68. Haasum Y, Fastbom J, Fratiglioni L, Kåreholt I, Johnell K. Pain treatment in elderly persons with and without dementia: a population-based study of institutionalized and home-dwelling elderly. Drugs Aging. 2011;28(4):283-293.

69. McCormick WC, Kukull WA, van Belle G, Bowen JD, Teri L, Larson EB. Symptom patterns and comorbidity in the early stages of Alzheimer's disease. JAm Geriatr Soc. 1994;42(5):517-521.

70. Shega JW, Paice JA, Rockwood K, Dale W. Is the presence of mild to moderate cognitive impairment associated with self-report of non-cancer pain? A cross-sectional analysis of a large population-based study. $J$ Pain Symptom Manage. 2010;39(4):734-742.

71. Scherder EJ, Slaets J, Deijen JB, et al. Pain assessment in patients with possible vascular dementia. Psychiatry. 2003;66(2): 133-145.

72. Husebo BS, Strand LI, Moe-Nilssen R, Borgehusebo S, Aarsland D, Ljunggren AE. Who suffers most? Dementia and pain in nursing home patients: a cross-sectional study. $J$ Am Med Dir Assoc. 2008;9(6): $427-433$
73. Morrison RS, Siu AL. A comparison of pain and its treatment in advanced dementia and cognitively intact patients with hip fracture. J Pain Symptom Manage. 2000;19(4):240-248.

74. Cornali C, Franzoni S, Gatti S, Trabucchi M. Diagnosis of chronic pain caused by osteoarthritis and prescription of analgesics in patients with cognitive impairment. J Am Med Dir Assoc. 2006;7(1):1-5.

75. Mäntyselkä P, Hartikainen S, Louhivuori-Laako K, Sulkava R. Effects of dementia on perceived daily pain in home-dwelling elderly people: a population-based study. Age Ageing. 2004;33(5):496-499.

76. Nygaard HA, Jarland M. Are nursing home patients with dementia diagnosis at increased risk for inadequate pain treatment? Int J Geriatr Psychiatry. 2005;20(8):730-737.

77. Scherder EJ, Bouma A. Is decreased use of analgesics in Alzheimer disease due to a change in the affective component of pain? Alzheimer Dis Assoc Disord. 1997;11(3):171-174.

78. Horgas AL, Elliott AF, Marsiske M. Pain assessment in persons with dementia: relationship between self-report and behavioral observation. J Am Geriatr Soc. 2009;57(1):126-132.

79. Lovheim H, Karlsson S, Gustafson Y. The use of central nervous system drugs and analgesics among very old people with and without dementia. Pharmacoepidemiol Drug Saf. 2008;17(9):912-918.

80. McLachlan AJ, Bath S, Naganathan V, et al. Clinical pharmacology of analgesic medicines in older people: impact of frailty and cognitive impairment. Br J Clin Pharmacol. 2011;71(3):351-364.

81. Barry HE, Parsons C, Passmore AP, Hughes CM. Community pharmacists and people with dementia: a cross-sectional survey exploring experiences, attitudes, and knowledge of pain and its management. Int J Geriatr Psychiatry. Epub January 24, 2013.

82. Husebo BS, Kunz M, Achterberg WP, et al. Pain assessment and treatment challenges in patients with dementia. Zeitschrift für Neuropsychologie. 2012;23(4):236-244.

83. Pieper MJ, van Dalen-Kok AH, Francke AL, et al. Interventions targeting pain or behaviour in dementia: A systematic review. Ageing Res Rev. Epub May 28, 2013.

84. Manfredi PL, Breuer B, Wallenstein S, Stegmann M, Bottomley G, Libow L. Opioid treatment for agitation in patients with advanced dementia. Int J Geriatr Psychiatry. 2003;18(8):700-705.

85. Buffum MD, Sands L, Miaskowski C, Brod M, Washburn A. A clinical trial of the effectiveness of regularly scheduled versus as-needed administration of acetaminophen in the management of discomfort in older adults with dementia. $J$ Am Geriatr Soc. 2004;52(7): 1093-1097.

86. Chibnall JT, Tait RC, Harman B, Luebbert RA. Effect of acetaminophen on behavior, well-being, and psychotropic medication use in nursing home residents with moderate-to-severe dementia. J Am Geriatr Soc. 2005;53(11):1921-1929.

87. Kovach CR, Logan BR, Noonan PE, et al. Effects of the Serial Trial Intervention on discomfort and behavior of nursing home residents with dementia. Am J Alzheimers Dis Other Demen. 2006;21(3): $147-155$.

88. Husebo BS, Ballard C, Sandvik R, Nilsen OB, Aarsland D. Efficacy of treating pain to reduce behavioural disturbances in residents of nursing homes with dementia: cluster randomised clinical trial. $B M J$. 2011;343:d4065.

89. Husebo BS, Ballard C, Cohen-Mansfield J, Seifert R, Aarsland D. The Response of Agitated Behavior to Pain Management in Persons with Dementia. Am J Geriatr Psychiatry. Epub April 20, 2013.

90. Cohen-Mansfield J, Jensen B. Assessment and treatment approaches for behavioral disturbances associated with dementia in the nursing home: self-reports of physicians' practices. J Am Med Dir Assoc. 2008;9(6):406-413.

91. Mokkink LB, Terwee CB, Gibbons E, et al. Inter-rater agreement and reliability of the COSMIN (COnsensus-based Standards for the selection of health status Measurement Instruments) checklist. BMC Med Res Methodol. 2010;10:82.

92. Cohen-Mansfield J, Lipson S. The utility of pain assessment for analgesic use in persons with dementia. Pain. 2008;134(1-2):16-23. 
93. Morello R, Jean A, Alix M, Sellin-Peres D, Fermanian J. A scale to measure pain in non-verbally communicating older patients: the EPCA-2 Study of its psychometric properties. Pain. 2007;133(1-3): $87-98$.
94. European Cooperation in Science and Technology (COST). Action TD1005: pain assessment in patients with impaired cognition, especially dementia [web page on the Internet]. Brussels: Available at: http://www. cost-td1005.net/. Accessed August 23, 2013.

\section{Publish your work in this journal}

Clinical Interventions in Aging is an international, peer-reviewed journal focusing on evidence-based reports on the value or lack thereof of treatments intended to prevent or delay the onset of maladaptive correlates of aging in human beings. This journal is indexed on PubMed Central, MedLine, the American Chemical Society's 'Chemical Abstracts

Service' (CAS), Scopus and the Elsevier Bibliographic databases. The manuscript management system is completely online and includes a very quick and fair peer-review system, which is all easy to use. Visit http://www.dovepress.com/testimonials.php to read real quotes from published authors.

Submit your manuscript here: http://www.dovepress.com/clinical-interventions-in-aging-journal 